\title{
Refractive index sensing with an air-slot photonic crystal nanocavity
}

\author{
Jana Jágerská,* Hua Zhang, Zhaolu Diao, Nicolas Le Thomas, and Romuald Houdré \\ Institut de Physique de la Matière Condensée, Ecole Polytechnique Fédérale de Lausanne (EPFL), \\ Station 3, CH-1015 Lausanne, Switzerland \\ *Corresponding author: jana.jagerska@epfl.ch
}

Received April 13, 2010; accepted June 2, 2010;

posted June 15, 2010 (Doc. ID 126913); published July 20, 2010

\begin{abstract}
We investigate an air-slot photonic crystal cavity for high-precision refractive index sensing. The high quality factor $\sim 2.6 \times 10^{4}$ of the cavity, along with a strong overlap between the resonant mode and the hollow core region, allows us to achieve an experimental sensitivity of $510 \mathrm{~nm}$ per refractive index unit (RUI) and a detection limit below $1 \times 10^{-5}$ RUI. The device has a remarkably low sensing volume of 40 aliters, holding less than $1 \times 10^{6}$ molecules. (C) 2010 Optical Society of America OCIS codes: $230.3120,230.5298,280.4788$.
\end{abstract}

Optical cavities realized in a photonic crystal (PhC) environment allow for both high quality factors $Q \sim 10^{6}$ and strong field confinement [1],2], resulting in enhanced light-matter interaction within an extremely small volume. They find a vast number of applications in nonlinear physics, quantum electrodynamics and, based on experimental demonstrations achieved to date, they also constitute a promising building block for optical sensors [圆, 四]. However, in typical PhC cavities, strong lightmatter interaction occurs in the high-index material where the majority of the optical field is located, making it difficult to efficiently probe the physical properties of analyte found within the hollow part of the cavity. This drawback can be overcome with the use of an air-slot cavity, i.e., a PhC cavity with a nanometer-wide slot designed in the cavity defect region [see Fig. [1(a)]. Because of a large electric field discontinuity at the slot boundaries [5], such a cavity can confine TE-polarized modes in the slot, thus allowing for a large interaction of the optical field with the low-index medium.

The air-slot cavity was first proposed in a onedimensional photonic crystal by Robinson et al. [6], followed by several different designs in PhC slabs [7, 8]. However, the full potential of the structure for sensitive on-chip detection has not yet been clearly assessed. In this Letter, we experimentally investigate a new type of a $\mathrm{PhC}$ air-slot cavity and demonstrate that it can act as a highprecision gas index sensor.

Figure 1(a) shows a typical air-slot PhC cavity, which is based on a W1 waveguide with an air-slot embedded in the line-defect region. To create a cavity, one can either modify the properties of the PhC mirror or the dimensions of the slot itself. The first approach was adopted by Di Falco et al. [7], who locally reduced the lattice constant of the photonic crystal to create a heterostructure cavity, as well as by Yamamoto et al. [8] and Gao et al. [9], where the holes adjacent to the slot were transversely shifted to form a width-modulated line-defect cavity. In the present design, the PhC lattice is kept unchanged, but the width of the air slot is locally reduced by $20 \mathrm{~nm}$ to delimit the volume of the cavity [insert of Fig. [1(a)] Reduced-slit width results in the formation of reflective barriers for the cavity mode, as can be inferred from the dispersion diagram of the slot $\mathrm{PhC}$ waveguides shown in
Fig. [1(b), When opening an air-slot inside the W1 defect region, the TE-polarized slot waveguide mode is lifted up from the dielectric band, shifting the mode cutoff to higher frequencies as the width of the slit increases. Consequently, the $120 \mathrm{~nm}$ slot waveguide mode lies at slightly higher frequencies than the $100 \mathrm{~nm}$ one, supporting a defect state when sandwiched between two $100 \mathrm{~nm}$ slot waveguide sections. The theoretical dispersion was calculated by the guided-mode expansion method [10] for a photonic crystal with a lattice constant $a=510 \mathrm{~nm}$ and a filling factor $f=0.4$.

We focus on a cavity with a length equal to three lattice constants $L=3 a$, the resonant state of which is found between the cutoffs of the 120 and $100 \mathrm{~nm}$ wide slot waveguides. The corresponding electric field distribution plotted in the inset of Fig. [1(b)] confirms that the cavity

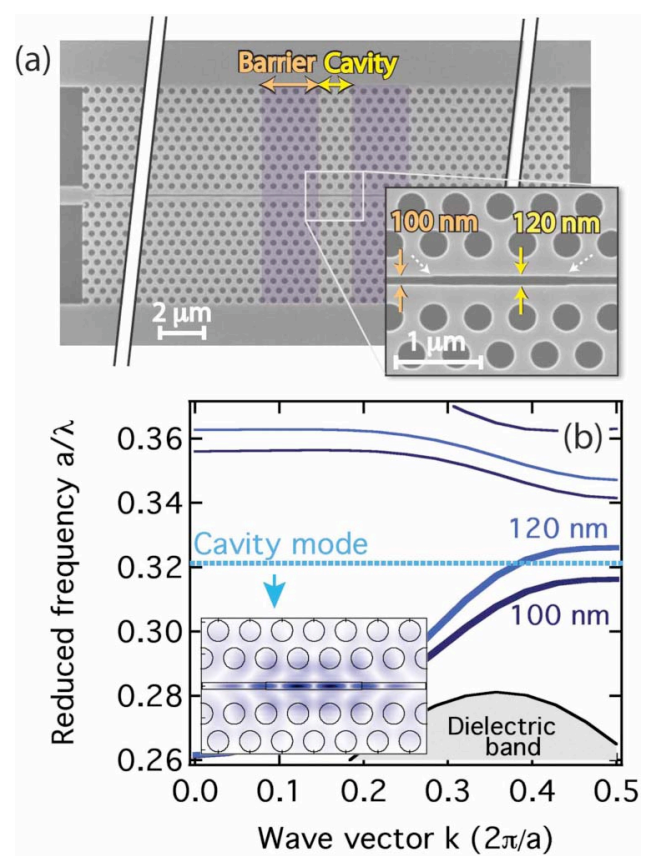

Fig. 1. (Color online) (a) Scanning electron microscopy image of the air-slot cavity. (b) Theoretical dispersion curves of the 100 and $120 \mathrm{~nm}$ PhC slot waveguides (solid line) shown together with the air-slot cavity resonance (dashed line). Inset: electric field distribution $\left|E_{x y}\right|$ of the cavity mode. 
mode is strongly confined within the slot region, having an effective mode volume [2] equal to $V_{\text {eff }}=0.05 \mu \mathrm{m}^{3}$ and the spatial overlap $\Gamma$ with the gas as high as 0.83 . Knowing the mode field overlap $\Gamma$, the first-order perturbation theory can be used to estimate the sensitivity $S$ of the device to the refractive index change $\Delta n$ inside the slot [11]:

$$
S=\frac{\Delta \lambda}{\Delta n}=\Gamma \frac{\lambda}{n_{\mathrm{eff}}}
$$

where $\lambda$ is the resonance wavelength of the cavity and $n_{\mathrm{eff}}^{2}=\Gamma n_{\mathrm{gas}}^{2}+(1-\Gamma) n_{\mathrm{Si}}^{2}$ is the effective index experienced by the cavity mode. For resonance frequency $\lambda=1570 \mathrm{~nm}, n_{\mathrm{gas}}=1$, and $n_{\mathrm{Si}}=3.47$, we get a sensitivity of $S=770 \mathrm{~nm}$ per refractive index unit (RIU), which is quite remarkable for a sensor with an active sensing volume as low as $0.04 \mu \mathrm{m}^{3}=40$ aliters.

The proposed air-slot cavity was processed on a $220 \mathrm{~nm}$ thick silicon-on-insulator wafer (SOI from Soitec, France) with a $2 \mu \mathrm{m}$ buried oxide layer. The ZEP-520A positive-tone electron-beam resist was used as the etching hard mask, and the transfer of the pattern from the ZEP to the Si core layer was performed in a single step, using inductively coupled plasma (ICP) etching with a gas combination of $\mathrm{SF}_{6}$ and $\mathrm{C}_{4} \mathrm{~F}_{8}$. The $2 \mu \mathrm{m}$ thick buried oxide layer was removed by etching for $30 \mathrm{~min}$ in fluoricbased acid to achieve a freestanding silicon membrane. Supercritical drying was performed to avoid strain in the membrane structure.

The fabricated device was characterized using a setup shown in Fig. 2(a), which combines the end-fire technique with optical imaging [12]. The air-slot cavity is excited using a TE-polarized, monochromatic diode laser tunable in the wavelength range of $1470-1660 \mathrm{~nm}$. The optical signal is coupled through a lensed fiber and adiabatically tapered access waveguides into the PhC structure and finally through a $5 a$ long barrier region into the cavity [see Fig. 1(a)]. The length of the barriers was carefully chosen to achieve a high loaded $Q$ factor while keeping the transmission at a detectable level. In parallel with the standard end-fire transmission measurement, the light emitted from the sample surface is collected by a high numerical aperture (NA $=0.8)$ microscope objective and detected with an infrared CCD camera. Direct imaging of the sample surface yields the real-space image, which allows us to monitor the optical field distribution at the cavity. Furthermore, by imaging the back-focal plane of the collecting lens, we can access the far-field image, which gives the angular emission spectrum of light radiated from the cavity.

The experimental transmission spectrum of the air-slot cavity is shown in Fig. 2(b), The cavity barriers are transparent down to $1590 \mathrm{~nm}$, where the mode cutoff of the $100 \mathrm{~nm}$ wide slot waveguide is found. Approximately $20 \mathrm{~nm}$ below this cutoff, we can observe a narrow transmission peak that corresponds to the resonant mode of the cavity. The spectral position of the cavity mode is unambiguously confirmed by the far-field imaging, which allows one to identify the cavity resonance although the transmitted signal is weak or accompanied with spurious resonances. The cavity mode exhibits a stable, highly symmetric far-field pattern [see the inset in Fig. 2(b)] that differs distinctly from the specklelike emission of the residual resonance peaks. A broad and weak resonance observed at $1580 \mathrm{~nm}$ between the cavity mode and the barrier cutoff was attributed to an evanescent mode, which can penetrate through the barriers due to evanescent coupling.

The light radiated from the sample surface and recorded as a function of the wavelength yields the emission spectrum of the cavity, which is shown in the inset of Fig. 2(b). It exhibits a better signal-to-noise ratio than the transmission and was, therefore, used to analyze the spectral properties of the cavity. The Lorentzian fit of the emission peak gives a linewidth of $60 \pm 1 \mathrm{pm}$, yielding a loaded quality factor $Q=26,000$.

To assess the sensing potential of the cavity, we expose the sample to gases of different refractive indices, such as air $(n=1.000265)$ [13], $\mathrm{N}_{2}(1.000270)$ [114], He (1.000032) [13], and $\mathrm{CO}_{2}$ (1.000407) [14]. The refractive index values are given at atmospheric pressure and were recalculated for $\lambda=1570 \mathrm{~nm}$ and room temperature $T=$ $20^{\circ} \mathrm{C}$ using the ideal gas model [15]. During the measurement, the sample is placed in a gas cell [see Fig. 2(a)] that is equipped with a gas inlet, $2 \mu \mathrm{m}$ size openings for the optical fibers, and an outlet for gas evacuation. The gas is injected into the cell at a constant rate, fills the volume of the cell, and escapes by the outlet opening, thus avoiding the overpressure inside the cell. The flux rate is set to a value just sufficient to entirely fill the cell volume with the test gas by observing the shift of the cavity resonance upon accruing concentration of the gas; when a new increase of the gas flux does not induce further shift in the
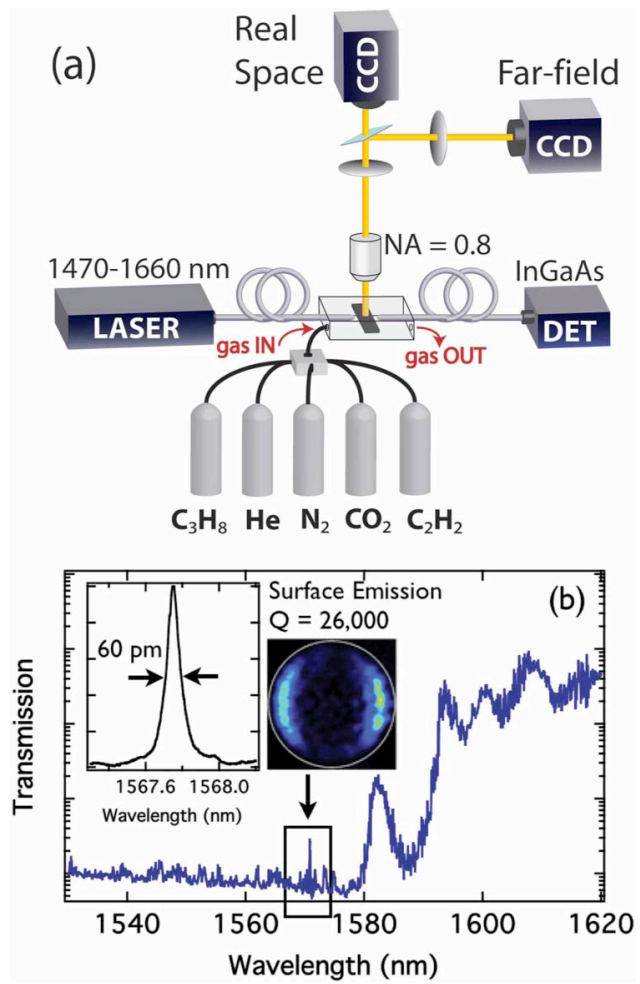

Fig. 2. (Color online) (a) Combined end-fire imaging setup. (b) Transmission spectrum of the air-slot cavity. Insets: surface emission spectrum (left) and the far-field image (right) at the cavity resonance. 

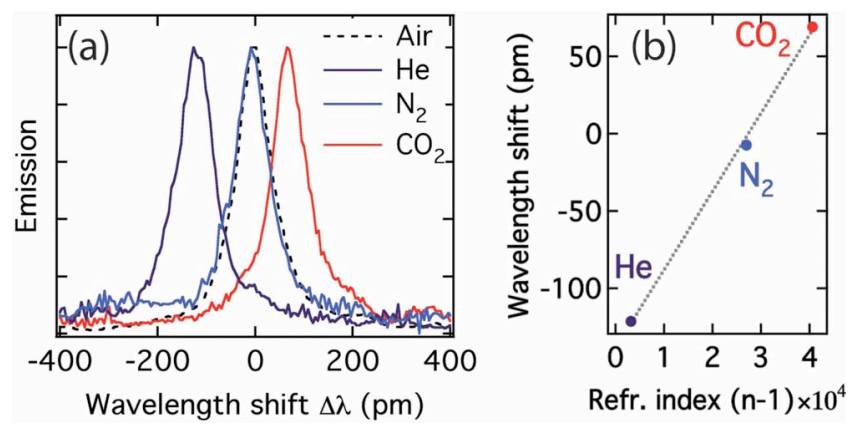

Fig. 3. (Color online) (a) Spectral position of the cavity resonance upon exposure to $\mathrm{He}, \mathrm{N}_{2}, \mathrm{CO}_{2}$, and the air. (b) Wavelength shift of the cavity resonance as a function of the refractive index $(n-1)$ of the respective gas.

resonance wavelength, the cell is considered to be completely filled with the analyte.

The shift of the air-slot cavity resonance in response to the change of the gas refractive index is shown in Fig. 6 . Considering air as the reference medium, we can observe both blue shift of the resonance peak for $\mathrm{He}$ and red shift for $\mathrm{CO}_{2}$. As plotted in Fig. 3(b), the dependence of the wavelength shift on the refractive index change $(n-1)$ is strictly linear and yields the experimental sensitivity $\Delta \lambda / \Delta n=510 \mathrm{~nm} / \mathrm{RIU}$, which is slightly lower than predicted by the numerical analysis. The small discrepancy can be attributed to structural differences between the modeled and the fabricated device. Repetitive measurements on a longer time scale show that the peak position can be retrieved with a precision of $\pm 3 \mathrm{pm}$, which implies the detection limit (defined as the ratio between the resolution and the sensitivity) of $1 \times 10^{-5}$ RIU. Nevertheless, it should be mentioned that along with the refractive index of the surrounding medium, there are other parameters that can induce a shift of the resonance frequency of the air-slot cavity mode, e.g., temperature, humidity adsorbed at the structure sidewalls, or progressive oxidation of the sample surface. In particular, the latter turns out to be the most detrimental, as it can induce a shift of the resonance peak by as much as $45 \mathrm{pm}$ per hour. This effect can be eliminated when working with $\mathrm{O}_{2}$-free gases, or compensated using an identical reference structure. Further enhancement of the sensor detection limit can be achieved by increasing the $Q$ factor of the cavity, which improves the precision of the resonance frequency readout. However, note that the 40 aliters sensing volume holds as little as $1 \times 10^{6}$ molecules; With the present sensitivity and resolution limit, the sensor is less than an order of magnitude away from the mesoscopic regime, where the effect of statistical fluctuations becomes significant and finally sets the physical limit of the sensor.

In conclusion, we have presented a highly sensitive refractive index detection scheme with a photonic crystal air-slot cavity. Because of a strong overlap of the cavity mode with the surrounding medium, such a cavity can achieve an experimental sensitivity of $510 \mathrm{~nm} / \mathrm{RIU}$ with the detection limit higher than $1 \times 10^{-5} \mathrm{RIU}$, which is comparable with state-of-the-art devices [3]. Yet, the major advantage of the sensor is an extremely small active sensing volume of 40 aliters, which is possible due to strong confinement of the optical field in $\mathrm{PhC}$ point-defect cavities. Such a small volume not only allows one to drastically decrease the necessary amount of analyte, but it also makes the sensor suitable for high-density integration oriented toward parallel or multiplex detection.

The authors acknowledge SOITEC S.A. for providing the SOI wafers and the financial support from the European projects ePIXnet (IST-004525), the MP0702 Action and the Swiss National Centre of Competence in Research Quantum Photonics.

\section{References}

1. E. Kuramochi, M. Notomi, S. Mitsugi, A. Shinya, T. Tanabe, and T. Watanabe, Appl. Phys. Lett. 88, 041112 (2006).

2. T. Asano, B. S. Song, Y. Akahane, and S. Noda, IEEE J. Sel. Top. Quantum Electron. 12, 1123 (2006).

3. X. Fan, I. M. White, S. I. Shopova, H. Zhu, J. D. Suter, and Y. Sun, Anal. Chim. Acta 620, 8 (2008).

4. M. Lončar, A. Scherer, and Y. Qiu, Appl. Phys. Lett. 82 , 4648 (2003).

5. W. Almeida, Q. X. Xu, C. A. Barrios, and M. Lipson, Opt. Lett. 29, 1209 (2004).

6. J. T. Robinson, C. Manolatou, L. Chen, and M. Lipson, Phys. Rev. Lett. 95, 143901 (2005).

7. A. Di Falco, L. O'Faolain, and T. F. Krauss, Appl. Phys. Lett. 94, 063503 (2009).

8. T. Yamamoto, M. Notomi, H. Taniyama, E. Kuramochi, Y. Yoshikava, Y. Torii, and T. Kuga, Opt. Express 16, 13809 (2008).

9. J. Gao, J. F. McMillan, M. C. Wu, J. J. Zheng, S. Assefa, and C. W. Wong, Appl. Phys. Lett. 96, 051123 (2010).

10. L. C. Andreani and D. Gerace, Phys. Rev. B 73, 235114 (2006).

11. N. A. Mortensen, S. Xiao, and J. H. Pedersen, Microfluid. Nanofluid. 4, 117 (2008).

12. N. Le Thomas, R. Houdré, M. V. Kotlyar, D. O'Brien, and T. F. Krauss, J. Opt. Soc. Am. B 24, 2964 (2007).

13. L'Air Liquide, Division Scientifique, Encyclopédie des Gaz (Elsevier, 1976).

14. A. C. Simmons, Opt. Commun. 25, 211 (1978).

15. Y. Clergent, C. Durou, and M. Laurens, J. Chem. Eng. Data 44, 197 (1999). 